\title{
Narcolepsy among Adults: A Descriptive Study
}

\author{
Nabat Arfi \& Dr. Shalini Agarwal \\ Department of Human Development \& Family Studies, School for Home Science, \\ Babasaheb Bhimrao Ambedkar University (A Central University) Lucknow, India
}

\begin{abstract}
Narcolepsy is a chronic disorder of impaired expression of wakefulness and rapid-eye-movement (REM) sleep, reported more in adults as they have less sleeping hours because of busy work schedules whether in office or at home. There are studies indicating narcolepsy among old people as they suffer from different diseases due to their age but studies showing narcoleptic adults are lacking. The purpose of present study is to know about this under-recognized disease during adulthood. Pathophysiological studies have shown that the disease is caused by the early loss of neurons in the hypothalamus that produce hypocretin, a wakefulnessassociated neurotransmitter present in cerebrospinal fluid. Only cataplexy exhibits a high specificity for diagnosis of narcolepsy. While the natural history is poorly defined, narcolepsy appears to be lifelong but not progressive. Mild disease severity, misdiagnoses or long delays in cataplexy expression often cause long intervals between symptom onset, presentation and diagnosis. Only 15-30\% of narcoleptic individuals are ever diagnosed or treated, and nearly half first present for diagnosis after the age of 40 years. Treatment includes counseling as to the chronic nature of narcolepsy, the potential for developing further symptoms reflective of REM sleep dyscontrol, and the hazards associated with driving and operating machinery. Non pharmacological management also includes maintenance of a strict wake-sleep schedule, good sleep hygiene, the benefits of afternoon naps and a programme of regular exercise. Thereafter, treatment is highly individualized, depending on the severity of daytime sleepiness, cataplexy and sleep disruption. It is important that general practitioners and other primary health-care workers identify abnormal daytime sleepiness early.
\end{abstract}

Keyword: sleeping disorder, cataplexy, narcolepsy, non-pharmacological therapy.

\section{Introduction}

Narcolepsy is a chronic disorder of the central nervous system characterized by the brain's inability to control sleep-wake cycles. At various times throughout the day, people with narcolepsy experience irresistible and sudden bouts of sleep, which can last from a few seconds to several minutes. In narcolepsy, sleep episodes can occur at any time. People may unwillingly fall asleep while at work or at school, when having a conversation, playing a game, eating a meal, or, most dangerously, when driving an automobile or operating other types of machinery. In addition to daytime sleepiness, other major symptoms include cataplexy (a sudden loss of voluntary muscle tone that may be triggered by strong emotions), vivid dream-like images or hallucinations during sleep onset or when waking, and brief episodes of total paralysis, also during sleep onset or when waking. The loss of muscle tone in cataplexy and sleep paralysis involves the simultaneous loss of both extensor reflexes (such as a knee tap and resulting leg jerk) and flexor reflexes (such as lifting the foot and/or leg following a foot prick or stepping on a sharp object). Normally, this kind of reflex loss exists only during REM sleep. Contrary to common beliefs, people with narcolepsy do not spend a substantially greater proportion of their time asleep during a 24-hour period than do normal sleepers. In addition to daytime drowsiness and uncontrollable sleep episodes, most individuals also experience poor sleep quality that can involve frequent awakenings during nighttime sleep, and other sleep disorders. For these reasons, narcolepsy is considered to be a disorder involving the loss of control of the normal boundaries between the sleeping and waking states.

For most adults, a normal night's sleep lasts about 8 hours and is composed of four to six separate sleep cycles. A sleep cycle is defined by a segment of non-rapid eye movement (NREM) sleep followed by a period of rapid eye movement (REM) sleep. The NREM segment can be further divided into increasingly deeper stages of sleep according to the size and frequency of brain waves. REM sleep is accompanied by bursts of rapid eye movement along with sharply heightened brain activity and temporary paralysis of the muscles that control posture and body movement. When subjects are awakened, they report that they were "having a dream" more often if they had been in REM sleep than if they had been in NREM sleep. Transitions from NREM to REM sleep are controlled by interactions among groups of neurons (nerve cells) located in different parts of the brain. Narcolepsy may have several causes. When cataplexy is present, narcolepsy is almost always caused by the lack of a brain neurotransmitter called hypocretin. Neurotransmitters are chemicals that neurons produce to communicate with each other and to regulate biological processes. Disruption typically arises from the death of 
approximately 70,000 brain cells containing hypocretin. Loss of hypocretin then results in an inability to regulate sleep. The normal organization of sleep is also changed. For normal sleepers a typical sleep cycle is about 100 to 110 minutes long, beginning with NREM sleep and transitioning to REM sleep after 80 to 100 minutes. People with narcolepsy frequently enter REM sleep within a few minutes of falling asleep. In cases without cataplexy, the disorder may be caused by a lack of hypocretin or by various other causes.

\section{Signs And Symptoms}

People with narcolepsy experience various types of day- and nighttime sleep problems that are associated with REM sleep disturbances that tend to begin subtly and may change dramatically over time.

- The most common major symptom, other than excessive daytime sleepiness (EDS), is cataplexy, which occurs in about 70 percent of all people with narcolepsy.

- Sleep paralysis and hallucinations are somewhat less common. Only 10 to 25 percent of affected individuals, however, display all four of these major symptoms during the course of their illness.

1. Excessive daytime sleepiness (EDS)-

- EDS, the symptom most consistently experienced by almost all individuals with narcolepsy, is usually the first to become clinically apparent.

- People with EDS describe it as a persistent sense of mental cloudiness, a lack of energy, a depressed mood, or extreme exhaustion.

- People tend to awaken from such unavoidable sleeps feeling refreshed and finding that their drowsiness and fatigue subsides for an hour or two.

2. Cataplexy-

- Cataplexy is a sudden loss of muscle tone that leads to feelings of weakness and a loss of voluntary muscle control.

- Attacks can occur at any time during the waking period, with individuals usually experiencing their first episodes several weeks or months after the onset of EDS.

- The most severe attacks result in a complete loss of tone in all voluntary muscles, leading to total physical collapse during which individuals are unable to move, speak, or keep their eyes open.

3. Sleep paralysis-

- The temporary inability to move or speak while falling asleep or waking also parallels REM-induced inhibitions of voluntary muscle activity.

- As with cataplexy, people remain fully conscious. sleep paralysis is frightening events, especially when first experienced.

- Shocked by suddenly being unable to move, many individuals fear that they may be permanently paralyzed or even dying.

4. Hallucinations-

- Hallucinations can accompany sleep paralysis or can occur in isolation when people are falling asleep or waking up.

- Hypnagogic hallucinations when occurring during sleep onset and as Hypnopompic hallucinations when occurring during waking, these images are unusually vivid and can be frightening.

- Most often, the content is primarily visual, but any of the other senses can be involved. These hallucinations represent another intrusion of an element of REM sleep — dreaming into the wakeful state.

5. Disrupted nocturnal sleep-

- While some individuals with narcolepsy have no difficulties falling asleep at night, most experience difficulties staying asleep.

- Sleep may be disrupted by insomnia, vivid dreaming, sleep talking, acting out while dreaming, and periodic leg movements.

\section{Causes}

When cataplexy is present, the cause most often is the discrete loss of brain cells that produce hypocretin. The reason for such cell loss remains unknown but appears to be autoimmune in nature. That is, the body's immune system selectively attacks hypocretin-containing brain cells. In extremely rare cases, narcolepsy is caused by a genetic defect that prevents normal production of hypocretin molecules. While researchers continue to seek out the root cause of narcolepsy, the general consensus is that your genetics, accompanied by an environmental trigger of some sort - a virus, for example - may affect your brain chemicals and cause narcolepsy. Although the cause of narcolepsy was not determined for many years after its discovery, scientists had discovered conditions that seemed to be associated with an increase in an individual's risk of having the disorder. Specifically, there appeared to be a strong link between narcoleptic individuals and certain genetic conditions. The neural control of normal sleep states and the relationship to narcolepsy are only partially understood. In 
humans, narcoleptic sleep is characterized by a tendency to go abruptly from a waking state to REM sleep with little or no intervening non-REM sleep.Scientists have discovered that people with narcolepsy are lacking in hypocretin (also called orexin), a chemical in the brain that activates arousal and regulates sleep. Narcoleptics generally do not have as many Hcrt cells (neurons that secrete hypocretin), which inhibits your ability to fully control your alertness and accounts for your tendency to fall asleep.

\section{Effects}

Some of the common effects of narcolepsy are:-

1. Physical well-being and safety - Many ordinary daily activities, such as driving, working, cooking, or walking, can become very dangerous if you fall asleep or lose muscle control unexpectedly.

2. Mental health - Narcolepsy can disrupt your life to the extent that it may lead to depression and anxiety.

3. Social and professional relationships - Unfortunately, sudden sleep episodes are often found humorous to those not familiar with narcolepsy. Some people may assume that you are lazy, rude, or even faking the sudden sleep episodes.

4. Intimate relationships - Your personal relationships, especially spousal relationships, can often suffer as a result of narcolepsy. Extreme sleepiness may also cause low sex drive and impotence.

5. Memory and attention - Narcolepsy may cause you to have problems remembering things.

\section{Diagnosis}

Diagnostic methods used to determine narcolepsy include:-

1. Epworth Sleepiness Scale:-Questionnaires are used to measure excessive sleepiness. The most commonly used questionnaire is the 8-question Epworth Sleepiness Scale (1991).

- Response to each question on a scale from 0 (not at all likely to fall asleep) to 3 (very likely to fall asleep) is obtained.

- $\quad$ The resulting total score is between 0 and 24 .

- Although what score constitutes abnormal sleepiness is controversial, total scores above 10 generally warrant investigation.

- You can take the Epworth Sleepiness Scale Test.

Epworth Sleepiness Scale to test for narcolepsy: The Epworth Sleepiness Scale measures daytime sleepiness. Use the following scale to choose the most appropriate number for each situation:

$0=$ would never doze or sleep.

$1=$ slight chance of dozing or sleeping

$2=$ moderate chance of dozing or sleeping

$3=$ high chance of dozing or sleeping

\section{Situation}

Chance of Dozing or Sleeping

Sitting and reading

Watching TV

Sitting inactive in a public place

Being a passenger in a motor vehicle for an hour or more

Lying down in the afternoon

Sitting and talking to someone

Sitting quietly after lunch (no alcohol)

Stopped for a few minutes in traffic while driving

Total score (add the scores up)

A total score of 10 or more is considered sleepy. A score of 18 or more is very sleepy. 
2. Polysomnography: - For this exam, one needs to come to a sleep laboratory about two hours prior to bedtime without making any changes in the daily habits. Then, the whole night's sleep is monitored and recorded. The following parameters are monitored:

- Electrical activity of the brain (electroencephalogram)

- Electrical activity of the heart (electrocardiogram)

- Movements of the muscles (electromyogram)

- Eye movements (electrooculogram)

- Respirations (oral thermistor or nasal pressure transducer)

These parameters are monitored as one passes through the various sleep stages (see Sleep: Understanding the Basics).If a person has narcolepsy, the polysomnograph shows short sleep latency of usually less than five minutes and an abnormally short latency prior to the first sleep onset REM sleep (SOREMPs). More than two SOREMPs and a mean sleep latency of less than five minutes strongly suggest narcolepsy.

3. Multiple Sleep Latency Test:-In this test, the time taken by a person to fall asleep (sleep latency) during the day while lying in a quiet room is measured. This test is performed the morning after the overnight polysomnogram. The person takes four or five scheduled naps every two hours. The first nap starts two hours after awakening that morning. People with normal sleep and alertness take about 10-20 minutes to fall asleep. Persons with narcolepsy (and other causes of abnormal sleepiness) take a much shorter time (less than five minutes) to go from wakefulness into sleep. Two weeks prior to these tests, the patient is asked to keep a sleep diary that records bedtime, wake-up times, and nap times. Their physician will inform them to gradually eliminate medications that could affect the sleep tests.

4. CSF (cerebrospinal fluid) hypocretin test:-This has not yet become part of the routine diagnostic tools for narcolepsy, but is being used more frequently. For this test a sample of cerebrospinal fluid is removed by lumbar puncture and sent to the laboratory for analysis. The specificity and sensitivity of the CSF hypocretin test are high enough that it should have clinical usefulness for diagnosis. Thus, defining the precise criteria for the diagnosis of narcolepsy and other sleep disorders is an evolving process.

\section{Treatment}

Patients with narcolepsy can be substantially helped, but not cure. Treatment is tailored to the individual, based on symptoms and therapeutic response. The time required to achieve optimal control of symptoms is highly variable, and may take several months or longer. Currently counseling, medication and lifestyle changes are used in combination as a narcolepsy treatment. These treatments while not curing the condition permanently will control the various symptoms thus allowing one to enjoy a full and free life.

\section{Lifestyle Changes:}

- Narcoleptics must avoid caffeine, alcohol and nicotine in all forms since they tend to interfere with one's ability to sleep properly.

- To schedule sleep periods during the day. In short narcoleptics must take a few pre planned naps for up to 20 minutes every day to ensure not sleep at awkward times.

- Talk to friends, employers and co-workers to ensure optimum help will be provided when needed.

- Regular exercise, since it will cause one to be alert during the day and sleep during the night.

- Maintain a healthy and balanced diet with all major food groups.

2. Medication: Common medications used to treat narcolepsy symptoms include:

- Stimulants. Stimulants are the mainstay of drug treatment for narcolepsy. These include modafinil (Provigil), a stimulant used during the day to promote wakefulness and alertness. Side effects of modafinil may include headache, nausea, dry mouth, and diarrhea. Psychiatric side effects, such as anxiety, mania, hallucinations, and suicidal thinking have also been reported, so the drug should be avoided if you have a history of depression, mania, or psychosis.

- Sodium oxybate (Xyrem). This strong drug may be prescribed if you have severe cataplexy. Sodium oxybate is also known as GHB, or the "date rape drug," but is considered safe for treating narcolepsy when used responsibly to promote sound sleep, diminish daytime sleepiness, and reduce incidences of cataplexy. However, the side effects can be serious and may include nausea, bed-wetting, and worsening of sleepwalking. Too high a dose can even lead to difficulty breathing, coma, and death.

- Antidepressants. Selective serotonin reuptake inhibitors (SSRIs) used to treat depression may also be used to help suppress REM sleep, and alleviate symptoms of cataplexy, hallucinations, and sleep paralysis. These include fluoxetine (Prozac), sertraline (Zoloft), and newer antidepressants such as venlafaxine (Effexor). 
While the most common side effects of antidepressants include decreased sexual desire, digestive problems, restlessness, headache, and insomnia, there can also be dangerous side effects.

\section{Counseling and Support Groups:}

- Sleeping at awkward times and places may be embarrassing or even cause depression, thus it is a must to join support groups and regularly visit a psychologist for professional counselling and help.

- When the above treatments are used in unison, it creates a powerful narcolepsy treatment which will allow narcoleptics to live a happy and relatively normal life.

Counseling has been given for various factors:-

How Much Sleep Do You Need? - Learn what happens when you're sleeping, how to determine your nightly sleep needs, and what you can do to bounce back from chronic sleep loss.

How to Sleep Better - The way you feel during the day hinges on how well you sleep at night. Find tips for getting better sleep and developing a regular sleep routine.

Sleep Disorders \& Sleeping Problems - When sleep problems are a regular occurrence, getting in the way of your daily routine and ability to function, you may be suffering from a sleep disorder.

Understanding Depression - Excessive sleepiness may be a sign of depression. Learning more about the signs, symptoms, causes, and treatment of depression.

Easy Exercise Tips - Exercise is just as good for the mind as it is for the body. Even a little regular exercise can boost your energy and mood and relieve stress, anxiety, and depression.

Senior Exercise and Fitness Tips - No matter your age, your health, or your fitness level, there are big and small ways to get more active and boost your energy and health.

How to Exercise with Limited Mobility - Even if injury, disability, illness, or weight problems have limited your mobility, you can still experience the benefits of exercise.

Healthy Eating - Learn easy ways to stick to a healthy diet to boost your energy, sharpen your memory, and stabilize your mood.

Stress Management - Manage stress by learning how to take charge of your thoughts, emotions, environment, and the way you deal with problems.

4. Antidepressant drug risk factors:

Antidepressants can produce unpleasant or dangerous side effects, including an increased risk of suicide. The risk factors are higher for:

a) People with bipolar disorder: Antidepressants can trigger mania or cause rapid cycling between depression and mania. (Note that many people with bipolar disorder are undiagnosed).

b) People over 65. SSRI medications may increase the risk for falls, fractures, and bone loss in older adults.

c) Pregnant women. The use of SSRI's late in pregnancy may lead to short-term withdrawal symptoms in newborns after delivery. Typical symptoms include tremor, restlessness, mild respiratory problems, and weak cry.

d) Teens, adolescents, and young adults. The U.S. Food and Drug Administration requires that all depression medications include a warning label about the increased risk of suicide in children and young adults.

5. Non Pharmacological Therapy: There are several non pharmacological intervention that may benefit the patient with narcolepsy:

- Avoiding certain drugs- some prescribed, over-the-counter, and illicit drugs can produce daytime sleepiness or insomnia. Such drugs should be avoided by patients with narcolepsy.

- Napping/sleep hygiene- behavioral interventions are often helpful maximal control of narcolepsy. One or two well-timed 20 minutes naps will often improve sleepiness for one to three hours.

- Psychosocial support- patient with narcolepsy face various psychosocial and work-related problems throughout their lives, as a result, they may have difficulty meeting economic and social responsibilities.

\section{Prognosis}

None of the currently available medications enables people with narcolepsy to consistently maintain a fully normal state of alertness. But EDS and cataplexy, the most disabling symptoms of the disorder, can be controlled in most patients with drug treatment. Often the treatment regimen is modified as symptoms change. Whatever the age of onset, patients find that the symptoms tend to get worse over the two to three decades after the first symptoms appear. Many older patients find that some daytime symptoms decrease in severity after age 60 . 


\section{References}

[1]. Narcolepsy, http://www.ninds.nih.gov/disorders/narcolepsy/detail_narcolepsy.htm. Retrieved 2010-08-05. Accessed on September 2012.

[2]. Narcolepsy,Adam.about.com. http://adam.about.com/reports/Narcolepsy.htm. Retrieved 2011-01-25. Accessed on September 2012.

[3]. Narcolepsy, http://www.ninds.nih.gov/disorders/narcolepsy/detail_narcolepsy.htm. Accessed on September 2012.

[4]. Sleep disorder, http://www.webmd.com/sleep-disorders/guide/narcolepsy. Accessed on October 2012

[5]. Treatments of Narcolepsy, http://www.helpguide.org/life/narcolepsy_symptom_causes_treatments.htm. Accessed on October 2012

[6]. Sleep problems, http://www.sleepfoundation.org/article/sleep-related-problems/narcolepsy-and-sleep, Accessed on October 2012.

[7]. Narcolepsy, http://health.nytimes.com/health/guides/disease/narcolepsy, Accessed on November 2012

[8]. Narcolepsy treatment, http://emedicine.medscape.com/article/1188433-treatment, Accessed on November 2012.

[9]. Prognosis of Narcolepsy, http://www.healthcentral.com/sleep-disorders/understanding-narcolepsy-000098_4-145.html, Accessed on November 2012.

[10]. Epworth Sleepiness Scale, http://www.sleepfoundation.org/sleep-scale, Accessed on November 2012 